\title{
ESTIMATION OF RIEMANNIAN BARYCENTRES
}

\section{HUILING LE}

\begin{abstract}
Using Jacobi field arguments, this paper describes an iterative procedure for finding the Riemannian barycentres of a class of probability measures on complete, simply connected Riemannian manifolds with a finite upper bound on their sectional curvatures. This, in particular, generalises an earlier result of the author's ('Locating Fréchet means with application to shape spaces', $A d v$. Appl. Probab. 33 (2001) 324-338).
\end{abstract}

\section{Introduction}

The concept of the Riemannian barycentre (or Riemannian centre of mass) of a probability measure on a Riemannian manifold has links with many different research areas. Among others, Karcher has used it to generalise the mollifier smoothing to maps between Riemannian manifolds [6]; Émery and Mokobodzki have linked it with convexity [3]; Jost has used it to provide a simplification of the variational theory [4]; Kendall has applied it to derive a fully probabilistic approach to the nonlinear elliptic variational theory of harmonic maps [7]; Oller and Corcuera have exploited it to give an intrinsic approach to statistical estimation based on the Fisher-Rao metric [12]; and Le has related it to the study of shape of the means in the statistical analysis of shape [10].

The three main questions concerning the Riemannian barycentre are: whether it exists at all for a given probability measure; if so, whether it is unique; and then how to estimate it. For a probability measure with bounded support, the Riemannian barycentre always exists. Assuming that such a barycentre does exist, an issue related to its location is considered in [2]. The uniqueness of the Riemannian barycentre inside the support of the probability measure has been addressed in, for example, [6] and [7]. The result of [7] is essentially the best possible; see also [8], which is devoted to a cleaner treatment of the Kendall result without the distractions of harmonic maps. It is easy to give examples where the Riemannian barycentre ceases to be unique when the condition given in [7] or [8] fails. The issue of the estimation of Riemannian barycentres has been studied in [11] in the case of locally symmetric Riemannian manifolds with non-negative sectional curvature, and in [9] in the context of the simplex shape spaces of non-positive sectional curvature. The result regarding estimation given in [11] is based on the construction of a contraction mapping using Jacobi fields. However, this results in a further unsatisfactory restriction on the radii of the balls supporting the probability measures under consideration.

The focus of the present paper is to improve on the result of [11], and to give an alternative estimation procedure. We show that, for any complete and simply connected Riemannian manifold whose sectional curvatures have a finite upper bound, as long as the (finite) radii of the balls supporting the probability measures satisfy the condition set in [7], the estimate that 
we propose here will always converge to the Riemannian barycentre inside the supporting ball. Note that we do not require the upper bound of the sectional curvatures to be positive. However, when the upper bound is non-positive, the corresponding Kendall result does not require the finiteness of the radii of the supporting balls that we have imposed.

Throughout this paper, we shall always assume that $\boldsymbol{M}$ is a complete and simply connected Riemannian manifold with Riemannian distance $d$, that its sectional curvatures have an upper bound $\kappa_{1}$ and that, if $\kappa_{1}>0$, it has injectivity $\operatorname{radius} \operatorname{inj}(\boldsymbol{M})$ (see [1, p. 95]). We shall use $r_{*}$ to denote $\frac{1}{2} \min \left\{\operatorname{inj}(\boldsymbol{M}), \pi / \sqrt{\kappa}_{1}\right\}$ if $\kappa_{1}>0$, or a given arbitrary positive constant if $\kappa_{1} \leqslant 0$.

\section{A Jacobi field estimate}

For simplicity, in and only in this section, we shall also assume that the sectional curvatures of $\boldsymbol{M}$ have a lower bound $\kappa_{0} \in \mathbb{R}$. That will not translate into a global bound in our applications, since there we shall only be working locally on a geodesic ball. Assuming such a lower bound $\kappa_{0}$, note that in the case when $\kappa_{1}>0$, if the dimension of $\boldsymbol{M}$ is even or if the dimension of $\boldsymbol{M}$ is odd and $\kappa_{0} \geqslant \kappa_{1} / 4$, then $\operatorname{inj}(\boldsymbol{M}) \geqslant \pi / \sqrt{\kappa}_{1}$ (see [1, pp. 98-100]) so that, in these cases, the constant $r_{*}$ defined above will be $\pi /\left(2 \sqrt{\kappa_{1}}\right)$.

The open (geodesic) ball

$$
\mathscr{B}\left(x_{0}, r\right)=\left\{x: d\left(x, x_{0}\right)<r\right\}
$$

in $\boldsymbol{M}$ is called convex if, for any $x, y \in \mathscr{B}\left(x_{0}, r\right)$, there is one and only one shortest geodesic from $x$ to $y$ in $\boldsymbol{M}$, and this geodesic lies in $\mathscr{B}\left(x_{0}, r\right)$. Note that the Riemannian distance $d$ is a smooth function with respect to either of its two variables when both of the variables are restricted to a convex ball. Note also that, for any $x_{0} \in \boldsymbol{M}$, the ball $\mathscr{B}\left(x_{0}, r\right)$ with radius $r \leqslant r_{*}$ is convex (see [1, p.103]).

The following lemma shows the influence of the lower sectional curvature bound on Jacobi fields; the result is complementary to a similar lower bound on Jacobi fields given in [5, p. 188], in terms of the upper sectional curvature bound.

Lemma 1. Let $\gamma:[0, \ell] \longrightarrow M$ be a unit speed geodesic, where $\ell<2 r_{*}$, and let $J$ be a Jacobi vector field along $\gamma$ such that $J(0)$ is tangent to $\dot{\gamma}(0)$. Then, for all $t \in[0, \ell]$,

$$
\left\langle\dot{J}^{\mathrm{nor}}(t), J(t)\right\rangle \leqslant \begin{cases}\sqrt{\kappa_{0}} \cot \left(\sqrt{\kappa_{0}} t\right)\left\|J^{\mathrm{nor}}(t)\right\|^{2}, & \kappa_{0}>0, \\ \frac{1}{t}\left\|J^{\mathrm{nor}}(t)\right\|^{2}, & \kappa_{0}=0, \\ \sqrt{-\kappa_{0}} \operatorname{coth}\left(\sqrt{-\kappa_{0}} t\right)\left\|J^{\mathrm{nor}}(t)\right\|^{2}, & \kappa_{0}<0,\end{cases}
$$

where $J^{\mathrm{nor}}(t)$ and $\dot{J}^{\mathrm{nor}}(t)$ denote the normal components, with respect to the geodesic $\gamma$ at $t$, of $J(t)$ and $\dot{J}(t)$ respectively.

Proof. We prove the lemma for the case when $\kappa_{0}>0$. The proof for the cases when $\kappa_{0} \leqslant 0$ is similar. Without loss of generality, we assume that $\langle\dot{\gamma}(0), \dot{J}(0)\rangle=\|J(0)\|=0$, and that $\dot{J}(0) \neq 0$, so that $J(t)$ is non-zero vector field and normal to $\dot{\gamma}(t)$ for all $t \in(0, \ell]$.

For a fixed $t_{0} \in(0, \ell]$, consider the Jacobi field $\tilde{J}_{t_{0}}(t)=J(t) /\left\|J\left(t_{0}\right)\right\|$ along $\gamma$. Then, since $\left\|\tilde{J}_{t_{0}}\left(t_{0}\right)\right\|=1$, and since

$$
\frac{\langle\dot{J}, J\rangle}{\|J\|^{2}}=\frac{\left\langle\dot{\tilde{J}}_{t_{0}}, \tilde{J}_{t_{0}}\right\rangle}{\left\|\tilde{J}_{t_{0}}\right\|^{2}}
$$


we have

$$
\begin{aligned}
\frac{\langle\dot{J}, J\rangle\left(t_{0}\right)}{\left\|J\left(t_{0}\right)\right\|^{2}} & =\left\langle\dot{\tilde{J}}_{t_{0}}, \tilde{J}_{t_{0}}\right\rangle\left(t_{0}\right) \\
& =\int_{0}^{t_{0}} \frac{d}{d t}\left\langle\dot{\tilde{J}}_{t_{0}}, \tilde{J}_{t_{0}}\right\rangle(t) d t \\
& =\int_{0}^{t_{0}}\left\{\left\langle\dot{\tilde{J}}_{t_{0}}, \dot{\tilde{J}}_{t_{0}}\right\rangle(t)+\left\langle\ddot{\tilde{J}}_{t_{0}}, \tilde{J}_{t_{0}}\right\rangle(t)\right\} d t \\
& =\int_{0}^{t_{0}}\left\{\left\langle\dot{\tilde{J}}_{t_{0}}, \dot{\tilde{J}}_{t_{0}}\right\rangle(t)-\left\langle R\left(\tilde{J}_{t_{0}}, \dot{\gamma}\right) \dot{\gamma}, \tilde{J}_{t_{0}}\right\rangle(t)\right\} d t
\end{aligned}
$$

where $R$ is the curvature tensor on $\boldsymbol{M}$.

For $t \in\left[0, t_{0}\right]$, write $E(t)$ for the parallel translate of $\tilde{J}_{t_{0}}\left(t_{0}\right)$ along $\gamma$ in the reverse direction to $\gamma(t)$, and let

$$
V(t)=\frac{\sin \left(\sqrt{\kappa_{0}} t\right)}{\sin \left(\sqrt{\kappa_{0}} t_{0}\right)} E(t) .
$$

Then, for $t \in\left[0, t_{0}\right], V$ is a vector field along $\gamma$ that satisfies the conditions $V(0)=\tilde{J}_{t_{0}}(0)=$ 0 and $V\left(t_{0}\right)=\tilde{J}_{t_{0}}\left(t_{0}\right)$. Since for no $t \in[0, \ell]$ is $\gamma(t)$ conjugate to $\gamma(0)$ along $\gamma$, it follows from the index lemma (see [1, p. 24]) that

$$
\begin{aligned}
\int_{0}^{t_{0}}\left\{\left\|\dot{\tilde{J}}_{t_{0}}(t)\right\|^{2}-\left\langle R\left(\tilde{J}_{t_{0}}, \dot{\gamma}\right) \dot{\gamma}, \tilde{J}_{t_{0}}\right\rangle(t)\right\} d t & \leqslant \int_{0}^{t_{0}}\left\{\|\dot{V}(t)\|^{2}-\langle R(V, \dot{\gamma}) \dot{\gamma}, V\rangle(t)\right\} d t \\
& \leqslant \int_{0}^{t_{0}}\left\{\|\dot{V}(t)\|^{2}-\kappa_{0}\|V(t)\|^{2}\right\} d t .
\end{aligned}
$$

Let $\boldsymbol{M}_{0}$ be a Riemannian manifold with the same dimension as that of $\boldsymbol{M}$ and with the constant sectional curvature $\kappa_{0}$, and let $\gamma_{0}$ be a unit speed geodesic on $\boldsymbol{M}_{0}$. Consider a Jacobi field $J_{0}$ along $\gamma_{0}$ that satisfies the boundary conditions

$$
\left\|J_{0}(0)\right\|=0, \quad\left\langle\dot{\gamma}_{0}(0), \dot{J}_{0}(0)\right\rangle=0, \quad \text { and } \quad\left\|\dot{J}_{0}(0)\right\|=\|\dot{J}(0)\| .
$$

Then, following a similar argument to that given above, we have

$$
\frac{\left\langle\dot{J}_{0}, J_{0}\right\rangle\left(t_{0}\right)}{\left\|J_{0}\left(t_{0}\right)\right\|^{2}}=\int_{0}^{t_{0}}\left\{\left\|\dot{\tilde{J}}_{0, t_{0}}(t)\right\|^{2}-\kappa_{0}\left\|\tilde{J}_{0, t_{0}}(t)\right\|^{2}\right\} d t,
$$

where $\tilde{J}_{0, t_{0}}(t)=J_{0}(t) /\left\|J_{0}\left(t_{0}\right)\right\|$.

However, since $\boldsymbol{M}_{0}$ has the constant sectional curvature $\kappa_{0}$, we know that $J_{0}$ must take the form

$$
J_{0}(t)=\frac{1}{\sqrt{\kappa_{0}}} \sin \left(\sqrt{\kappa_{0}} t\right) E_{0}(t),
$$

where $E_{0}$ is the vector field of the parallel translation of $\dot{J}_{0}(0)$ along $\gamma_{0}$ (see [5, p. 187]). Thus, for all $t \in\left[0, t_{0}\right]$,

$$
\left\|\dot{\tilde{J}}_{0, t_{0}}(t)\right\|=\|\dot{V}(t)\| \quad \text { and } \quad\left\|\tilde{J}_{0, t_{0}}(t)\right\|=\|V(t)\| .
$$

The required result then follows from (1), (2), (3) and the fact that

$$
\frac{\left\langle\dot{J}_{0}, J_{0}\right\rangle\left(t_{0}\right)}{\left\|J_{0}\left(t_{0}\right)\right\|^{2}}=\sqrt{\kappa_{0}} \cot \left(\sqrt{\kappa_{0}} t_{0}\right) \text {. }
$$


Note that, if $\gamma$ is a geodesic $[0,1] \longrightarrow M$ with speed $\|\dot{\gamma}\|<2 r_{*}$ and $J$ is a Jacobi field along $\gamma$, then $\gamma(t /\|\dot{\gamma}\|)$ is a unit speed geodesic $[0,\|\dot{\gamma}\|] \longrightarrow \boldsymbol{M}$ and $J(t /\|\dot{\gamma}\|)$ is a Jacobi field along $\gamma(t /\|\dot{\gamma}\|)$. Thus, the result of Lemma 1 for the estimate for $J(t /\|\dot{\gamma}\|)$ in particular gives, for $t \in[0,1]$,

$$
\left\langle\dot{J}^{\text {nor }}(t), J(t)\right\rangle \leqslant \begin{cases}\|\dot{\gamma}\| \sqrt{\kappa_{0}} \cot \left(\|\dot{\gamma}\| \sqrt{\kappa_{0}} t\right)\left\|J^{\text {nor }}(t)\right\|^{2}, & \kappa_{0}>0, \\ \frac{1}{t}\left\|J^{\text {nor }}(t)\right\|^{2}, & \kappa_{0}=0, \\ \|\dot{\gamma}\| \sqrt{-\kappa_{0}} \operatorname{coth}\left(\|\dot{\gamma}\| \sqrt{-\kappa_{0}} t\right)\left\|J^{\text {nor }}(t)\right\|^{2}, & \kappa_{0}<0 .\end{cases}
$$

Since $\theta \cot (\theta) \leqslant 1$ for $\theta \in[0, \pi)$, we have the following result.

Corollary. Suppose that $\gamma:[0,1] \longrightarrow M$ is a geodesic with $\|\dot{\gamma}\|<2 r_{*}$, and let $J$ be a Jacobi vector field along $\gamma$ such that $J(0)$ is tangent to $\dot{\gamma}(0)$. Then

$$
\left\langle\dot{J}^{\text {nor }}(1), J(1)\right\rangle \leqslant c\left(r_{*}, \kappa_{0}\right)\left\|J^{\text {nor }}(1)\right\|^{2},
$$

where

$$
c\left(r_{*}, \kappa_{0}\right)= \begin{cases}1, & \kappa_{0} \geqslant 0, \\ 2 r_{*} \sqrt{-\kappa_{0}} \operatorname{coth}\left(2 r_{*} \sqrt{-\kappa_{0}}\right), & \kappa_{0}<0 .\end{cases}
$$

Note that, since $\theta \operatorname{coth}(\theta) \geqslant 1$ for $\theta \geqslant 0$, we always have $c\left(r_{*}, \kappa_{0}\right) \geqslant 1$. Note also that $\theta \operatorname{coth}(\theta)$ is an increasing function that is unbounded above for $\theta \geqslant 0$.

\section{Finding the Riemannian barycentre}

Suppose that $\mu$ is a probability measure on $\boldsymbol{M}$, and define

$$
F_{\mu}: M \longrightarrow \mathbb{R}_{+} ; \quad x \longmapsto \frac{1}{2} \int_{M} d(x, y)^{2} d \mu(y) .
$$

Then a point $\hat{x} \in \boldsymbol{M}$ is a Riemannian barycentre of $\mu$ if and only if the energy functional $F_{\mu}$ achieves its local minimum at $\hat{x}$.

The following result is due to W. S. Kendall; the uniqueness clauses are given by [7, Theorem 7.3] and the characterisation is a direct consequence of [7, proof of Lemma 7.2].

LEMMA 2. (i) If $\kappa_{1}>0$ and if the support of $\mu$ is contained in the ball $\mathcal{B}\left(x_{0}, r\right)$ with $r \leqslant r_{*}$, then the Riemannian barycentre $\hat{x}$ of $\mu$ in $\mathcal{B}\left(x_{0}, r\right)$ is unique.

(ii) If $\kappa_{1} \leqslant 0$ and if $F_{\mu}$ is finite at a point of $\boldsymbol{M}$, then the Riemannian barycentre $\hat{x}$ of $\mu$ is always unique.

In either case, the barycentre is characterised by $\operatorname{grad} F_{\mu}(\hat{x})=0$.

This result is a generalisation of an earlier result of Karcher [6], where the same conclusion holds under the more restrictive requirement that $r<r_{*} / 2$. Note that it is shown in [11] that, under Karcher's condition, $F_{\mu}$ actually achieves its global minimum at the Riemannian barycentre $\hat{x}$ of $\mu$ in $\mathscr{B}\left(x_{0}, r\right)$.

The main result of this paper is an estimate of the Riemannian barycentre $\hat{x}$ under the conditions set in Lemma 2, with the further restriction that the support of $\mu$ be contained in a finite ball $\mathscr{B}\left(x_{0}, r\right)$ when $\kappa_{1} \leqslant 0$. To describe our results, we now write $\kappa_{0}$ for the lower bound of the sectional curvatures in $\overline{\mathcal{B}\left(x_{0}, r\right)}$. Since $r$ is finite, $\kappa_{0}$ is also finite. 


\section{Estimation of Riemannian barycentres}

We first look at the behaviour of $F_{\mu}$ along a geodesic.

Lemma 3. If the support of $\mu$ is contained in the ball $\mathcal{B}\left(x_{0}, r_{*}\right)$ then, along any geodesic $\gamma:[0,1] \longrightarrow \mathcal{B}\left(x_{0}, r_{*}\right)$

$$
\frac{d^{2}}{d t^{2}} F_{\mu}(\gamma(t)) \leqslant c\left(r_{*}, \kappa_{0}\right)\|\dot{\gamma}(t)\|^{2},
$$

where the constant $c\left(r_{*}, \kappa_{0}\right) \geqslant 1$ is given by (4).

Proof. Consider the family of geodesics from $y$ to $\gamma(t): c_{y}(s, t)=\exp _{y}\left\{s \exp _{y}^{-1}(\gamma(t))\right\}$. Since, for any $x, y \in \mathscr{B}\left(x_{0}, r_{*}\right)$, there is a unique shortest geodesic between them that lies entirely in $\mathscr{B}\left(x_{0}, r_{*}\right)$, it follows that there is a unique $v \in \mathcal{T}_{y}(\boldsymbol{M})$ such that $\exp _{y}^{-1}(x)=v$, where $\mathcal{T}_{y}(\boldsymbol{M})$ denotes the tangent space of $\boldsymbol{M}$ at $y$ and $\exp _{y}$ is the Riemannian exponential map of $\boldsymbol{M}$ at $y$. Hence the geodesic $c_{y}(\cdot, t)$ is well defined. Note also that, for each $t \in[0,1]$, the geodesic $c_{y}(\cdot, t)$ lies entirely in $\mathscr{B}\left(x_{0}, r_{*}\right)$ with total length less than $2 r_{*}$.

It is shown in [6] that

$$
\frac{d^{2}}{d t^{2}} F_{\mu}(\gamma(t))=\int_{M}\left\langle\dot{\gamma}(t),\left.\frac{D}{d s} \frac{d c_{y}(s, t)}{d t}\right|_{s=1}\right\rangle d \mu(y) .
$$

If we denote the Jacobi field $d c_{y}(s, t) / d t$ along $s \longrightarrow c_{y}(s, t)$ by $J(s)$, then $J(0)=0$, $J(1)=\dot{\gamma}(t)$ independent of $y$ and

$$
\dot{J}(1)=\left.\frac{D}{d s} \frac{d c_{y}(s, t)}{d t}\right|_{s=1} .
$$

On the other hand, since $j^{\tan }$ is a tangential Jacobi field along $s \longrightarrow c_{y}(s, t)$ with $J^{\tan }(0)=$ 0 , we have $j^{\tan }(1)=J^{\tan }(1)$ (see [5, p. 172]). Thus we see, by the corollary to Lemma 1 , that

$$
\begin{aligned}
\frac{d^{2}}{d t^{2}} F_{\mu}(\gamma(t)) & =\int_{M}\langle J(1), \dot{J}(1)\rangle d \mu(y) \\
& =\int_{M}\left\{\left\langle J(1), \dot{J}^{\text {nor }}(1)\right\rangle+\left\langle J(1), \dot{J}^{\tan }(1)\right\rangle\right\} d \mu(y) \\
& \leqslant \int_{M}\left\{\left\|J(1)^{\mathrm{nor}}\right\|^{2}+\left\|J(1)^{\tan }\right\|^{2}\right\} d \mu(y) \\
& =c\left(r_{*}, \kappa_{0}\right)\|\dot{\gamma}(t)\|^{2},
\end{aligned}
$$

as required.

Since $r_{*}$ is at most half the injectivity radius, it follows that there is a unique

$$
v_{y} \in \exp _{x}^{-1}\left(\mathscr{B}\left(x_{0}, r_{*}\right)\right) \cap \mathbf{B}_{x}\left(2 r_{*}\right)
$$

such that $\exp _{x}^{-1}(y)=v_{y}$, where $\mathbf{B}_{x}\left(2 r_{*}\right)=\left\{v \in \mathcal{T}_{x}(\boldsymbol{M}):|v|<2 r_{*}\right\}$. Note that $\left|v_{y}\right|=$ $d(x, y)$. Thus, if the support of $\mu$ is contained in the ball $\mathscr{B}\left(x_{0}, r_{*}\right)$, we have, for any $x \in \mathscr{B}\left(x_{0}, r_{*}\right)$,

$$
\begin{aligned}
\operatorname{grad} F_{\mu}(x) & =-\int_{\boldsymbol{M}} \exp _{x}^{-1}(y) d \mu(y) \\
& =-\int_{\exp _{x}^{-1}\left(\mathscr{B}\left(x_{0}, r_{*}\right)\right) \cap \mathbf{B}_{x}\left(2 r_{*}\right)} v d\left(\mu \circ \exp _{x}\right)(v)
\end{aligned}
$$


Extending $\mu \circ \exp _{x}$ to the entire tangent space $\mathcal{T}_{x}(\boldsymbol{M})$ by defining

$$
\mu \circ \exp _{x}\left\{\mathcal{T}_{x}(\boldsymbol{M}) \backslash\left(\exp _{x}^{-1}\left(\mathscr{B}\left(x_{0}, r_{*}\right)\right) \cap \mathbf{B}_{x}\left(2 r_{*}\right)\right)\right\}=0
$$

we can write

$$
\operatorname{grad} F_{\mu}(x)=-\int_{\mathcal{T}_{x}(\boldsymbol{M})} v d\left(\mu \circ \exp _{x}\right)(v)
$$

and, clearly, the right-hand expression in (6) is the negative of the Euclidean mean of the induced probability distribution $\mu \circ \exp _{x}$ on $\mathcal{T}_{x}(\boldsymbol{M})$.

For $x \in \mathscr{B}\left(x_{0}, r_{*}\right)$, denote by $\gamma_{x}(t)$ the geodesic

$$
\gamma_{x}(t)=\exp _{x}\left(-t \operatorname{grad} F_{\mu}(x)\right), \quad t \in \mathbb{R} .
$$

The following lemma shows that the restriction of $F_{\mu}$ to $\gamma_{x}$ decreases over an interval $\left[0, t_{x}\right]$, where $t_{x} \leqslant 1 /\left(2 c\left(r_{*}, \kappa_{0}\right)\right)$. It also gives a lower bound for the absolute value of that decrease.

Lemma 4. Suppose that the support of $\mu$ is contained in the ball $\mathcal{B}\left(x_{0}, r_{*}\right)$. Then, for each $x \in \overline{\mathcal{B}\left(x_{0}, r_{*}\right)}$, there exists a $t_{x} \in\left(0,1 /\left(2 c\left(r_{*}, \kappa_{0}\right)\right)\right]$ such that, if $\operatorname{grad} F_{\mu}(x) \neq 0, F_{\mu}\left(\gamma_{x}(t)\right)$ is decreasing on $\left(0, t_{x}\right]$ and

$$
F_{\mu}(x)-F_{\mu}\left(\gamma_{x}(t)\right) \geqslant \frac{t}{2}\left\|\operatorname{grad} F_{\mu}(x)\right\|^{2}, \quad t \in\left(0, t_{x}\right] .
$$

Proof. For each $x \in \overline{\mathcal{B}\left(x_{0}, r_{*}\right)}$, let

$$
t_{x}=\frac{1}{2 c\left(r_{*}, \kappa_{0}\right)} \sup \left\{t \in[0,1]: \gamma_{x}(t) \in \overline{\mathcal{B}\left(x_{0}, r_{*}\right)}\right\} \text {. }
$$

Note that, for any $x \in \partial \mathscr{B}\left(x_{0}, r_{*}\right), \operatorname{grad} F_{\mu}(x)$ is transverse to $\partial \mathscr{B}\left(x_{0}, r_{*}\right)$ by $(5)$, and hence

$$
t_{x}>0, \quad \text { for all } x \in \overline{\mathcal{B}\left(x_{0}, r_{*}\right)} \text {. }
$$

Then $t_{x} \in\left(0,1 /\left(2 c\left(r_{*}, \kappa_{0}\right)\right)\right]$, and, if $\operatorname{grad} F_{\mu}(x)=0$, then $\gamma_{x}(t)=x$ for all $t$, and so $t_{x}=1 /\left(2 c\left(r_{*}, \kappa_{0}\right)\right)$.

If $\operatorname{grad} F_{\mu}(x) \neq 0$, then it follows from

$$
\left.\frac{d \gamma_{x}(t)}{d t}\right|_{t=0}=-\operatorname{grad} F_{\mu}(x)
$$

that

$$
\left.\frac{d F_{\mu}\left(\gamma_{x}(t)\right)}{d t}\right|_{t=0}=\left\langle\operatorname{grad} F_{\mu}(x),\left.\frac{d \gamma_{x}(t)}{d t}\right|_{t=0}\right\rangle=-\left\|\operatorname{grad} F_{\mu}(x)\right\|^{2}<0 .
$$

On the other hand, since $\gamma_{x}(t)$ lies in $\mathcal{B}\left(x_{0}, r_{*}\right)$ for $t \in\left[0, t_{x}\right]$, we see, by Lemma 3 , that

$$
\frac{d^{2} F_{\mu}\left(\gamma_{x}(t)\right)}{d t^{2}} \leqslant c\left(r_{*}, \kappa_{0}\right)\left\|\operatorname{grad} F_{\mu}(x)\right\|^{2}, \quad t \in\left[0, t_{x}\right] .
$$

This, together with (9), implies that, for $t \in\left(0, t_{x}\right]$,

$$
\begin{aligned}
\frac{d F_{\mu}\left(\gamma_{x}(t)\right)}{d t} & \leqslant-\left\|\operatorname{grad} F_{\mu}(x)\right\|^{2}+c\left(r_{*}, \kappa_{0}\right)\left\|\operatorname{grad} F_{\mu}(x)\right\|^{2} t \\
& \leqslant-\frac{1}{2}\left\|\operatorname{grad} F_{\mu}(x)\right\|^{2}<0,
\end{aligned}
$$

and so, in particular, $F_{\mu}\left(\gamma_{x}(t)\right)$ is decreasing on $\left(0, t_{x}\right]$. The result (8) is a consequence of (10) together with the mean-value theorem. 
We are now in a position to establish the main result of the paper.

Suppose that the support of $\mu$ is contained in the ball $\mathcal{B}\left(x_{0}, r_{*}\right)$. For a given $x \in$ $\overline{\mathscr{B}\left(x_{0}, r_{*}\right)}$, we now define a sequence of points $\left\{x_{n}: n \geqslant 0\right\}$ in $\boldsymbol{M}$ as follows:

$$
x_{0}=x \quad \text { and } \quad x_{n+1}=\exp _{x_{n}}\left(-t_{x_{n}} \operatorname{grad} F_{\mu}\left(x_{n}\right)\right), \quad \text { for } n \geqslant 0 .
$$

THEOREM. Suppose that the support of $\mu$ is contained in the ball $\mathcal{B}\left(x_{0}, r_{*}\right)$, and that $\left\{x_{n}: n \geqslant 0\right\}$ are defined as above. Then, for any $x \in \overline{\mathcal{B}\left(x_{0}, r_{*}\right)}$, the sequence $\left\{x_{n}: n \geqslant 0\right\}$ defined above converges to the Riemmanian barycentre $\hat{x}$ of $\mu$ in $\mathscr{B}\left(x_{0}, r_{*}\right)$ as $n \rightarrow \infty$.

Note that the theorem generalises the result given in [11] on the estimation of Riemmanian barycentres, which was obtained using a different method.

Proof. Suppose first that, for all $n>0, \operatorname{grad} F_{\mu}\left(x_{n}\right) \neq 0$. Then, by Lemma 4,

$$
F_{\mu}\left(x_{n}\right)>F_{\mu}\left(x_{n+1}\right) \text {. }
$$

Equation (11) shows that the sequence $F_{\mu}\left(x_{n}\right), n \geqslant 0$, is a strictly decreasing sequence that is also bounded below by zero, so that $\lim _{n \rightarrow \infty} F_{\mu}\left(x_{n}\right)$ exists.

Since the initial conditions for $\gamma_{x}$ vary smoothly with $x, t_{x}$ is continuous in $x$, so we find that $t_{*}=\inf \left\{t_{x}: x \in \overline{\mathcal{B}\left(x_{0}, r_{*}\right)}\right\}$ is strictly positive and that, by Lemma 3 ,

$$
F_{\mu}\left(x_{n}\right)-F_{\mu}\left(x_{n+1}\right) \geqslant \frac{t_{*}}{2}\left\|\operatorname{grad} F_{\mu}\left(x_{n}\right)\right\|^{2} .
$$

Hence the convergence of $\left\{F_{\mu}\left(x_{n}\right): n \geqslant 0\right\}$ implies that

$$
\lim _{n \rightarrow \infty}\left\|\operatorname{grad} F_{\mu}\left(x_{n}\right)\right\|=0 .
$$

Since $\left\{x_{n}: n \geqslant 0\right\}$ is contained in $\overline{\mathscr{B}\left(x_{0}, r_{*}\right)}$ and so is a bounded sequence, there is a convergent subsequence with limit $\tilde{x}$ in the ball. It is clear that $\operatorname{grad} F_{\mu}(\tilde{x})=0$ and so, by Lemma 2, we must have $\tilde{x}=\hat{x}$. It then follows that $\hat{x}$ is the only possible limit of any convergent subsequence of $\left\{x_{n}: n \geqslant 0\right\}$. This in turn implies that $\left\{x_{n}: n \geqslant 0\right\}$ itself converges to $\hat{x}$, since otherwise we could find a subsequence that converges to a limit other than $\hat{x}$, contradicting the above conclusion.

If there is an $n_{0}>0$ such that $\operatorname{grad} F_{\mu}\left(x_{n_{0}}\right)=0$, then $x_{n_{0}}=\hat{x}$, and it is easy to see that, for all $n>n_{0}, x_{n}=x_{n_{0}}$.

Acknowledgement. I am grateful to an anonymous referee for comments that have guided me to a stronger version of the results.

\section{References}

1. J. Cheeger and D. G. EBIN, Comparison theorems in Riemannian geometry (North Holland, Amsterdam, 1975). 194, 195

2. J. M. CoRcuera and W. S. Kendall, 'Riemannian barycentres and geodesic convexity', Math. Proc. Camb. Phil. Soc. 127 (1999) 253-269. 193

3. M. Émery and G. MokobodzKI, 'Sur le barycentre d'une probabilité dans une variéte', Séminaire Probabilite XXV, Lect. Notes in Math. 1485 (Springer, New York, 1991) 220-233. 193

4. J. Jost, 'Equilibrium maps between metric spaces', Calc. Var. 2 (1994) 173-204. 193 
5. J. Jost, Riemannian geometry and geometric analysis (Springer, Berlin, 1998). 194, 195,197

6. H. KARCHER, 'Riemannian center of mass and mollifier smoothing', Comm. Pure Appl. Math. 30 (1977) 509-541. 193, 196, 197

7. W. S. Kendall, 'Probability, convexity, and harmonic maps with small image I: uniqueness and fine existence', Proc. London Math. Soc. 61 (1990) 371-406. 193, 196

8. W. S. Kendall 'Convexity and the hemisphere', J. London Math. Soc. 43 (1991) 567-576. 193

9. A. Kume and H. Le, 'On Fréchet means in simplex shape spaces', Adv. Appl. Prob. 35 (2003) 885-897. 193

10. H. LE, 'Mean size-and-shapes and mean shapes: a geometric point of view', Adv. Appl. Probab. 27 (1995) 44-55. 193

11. H. LE, 'Locating Fréchet means with application to shape spaces', Adv. Appl. Probab. 33 (2001) 324-338. 193, 196, 199

12. J. M. Oller and J. M. Corcuera, 'Intrinsic analysis of the statistical estimation', Ann. Statist. 23 (1995) 1562-1581. 193

Huiling Le Huiling.Le@nottingham.ac.uk

http://www.maths.nottingham.ac.uk/htbin-local/staff.info?hl

School of Mathematical Sciences

University of Nottingham

University Park

Nottingham, NG7 2RD

United Kingdom 